\title{
EFFECTS OF COGNITIVE TASKS ON DRIVERS' EYE BEHAVIOR AND PERFORMANCE
}

\author{
Xianjun Sam Zheng, Yu-chi Tai and George W. McConkie \\ Educational Psychology and Beckman Institute \\ University of Illinois at Urbana-Champaign \\ 405 North Mathews Avenue \\ Urbana, Illinois USA \\ E-mail: gmcconk@uiuc.edu
}

\begin{abstract}
Safe driving involves obtaining and using required visual information. Recent studies have shown that this information acquisition is compromised as a driver performs other mental tasks. We conducted an experiment, inspired by Recarte and Nunes (2000), to investigate the effect of cognitive tasks on drivers' eye behavior and performance in a single monitor, PC-based driving simulator. The eye behavior (i.e., gaze direction and duration) and driving performance (i.e., lane keeping and speed control) of twelve college students were recorded as they drove in three environments (i.e., highway, rural, urban) under three secondary task conditions (none, verbal task, spatial-imagery task). The results confirmed Recarte and Nunes (2000) observation that such tasks greatly reduce the time and frequency of such safety-related behaviors as checking the speedometer and rear view mirrors, with the spatial-imagery task having the largest effect. Pupil diameter increases significantly when performing secondary tasks, confirming the usefulness of that measure as an indicator of processing load. In contrast, these secondary tasks have no effect on lane-keeping accuracy, though they do increase variation in speed. From the perspective of multiple resource theory (Wickens, 2002; Wickens \& Hollands, 2000), this suggests that lane keeping, speed control and other safety-monitoring activities (i.e., checking mirrors and speedometer) all require attentional resources, and that when resources must be given to some new task, there is a prioritization of the remaining tasks. Lane-keeping, the failure of which would produce the most apparent driving failures, is given the highest priority, with other safetymonitoring activities given lower priority. The method employed here can be used to examine the effect of driver activities and devices on their monitoring of safety-related information.
\end{abstract}

